\title{
Grundlagen des chilenischen Umweltrechts
}

\author{
Von Jorge Bermúdez, Valparaíso/Gießen*
}

\section{Einleitung}

Die Entwicklung des chilenischen Umweltrechts kann in zwei Phasen unterteilt werden. In einer ersten Phase bestanden nur die sog. zufälligen oder akzidentiellen umweltrechtlichen Normen. Das Umweltrecht in diesem Sinne erfasste also alle Regelungen, die eine Auswirkung auf den Umweltschutz hatten, die aber vom Gesetzgeber bzw. der Verwaltung für ein anderes Ziel vorgesehen waren ${ }^{1}$. Als Beginn der zweiten Phase der Entwicklung des chilenischen Umweltrechts gilt das Inkrafttreten der Verfassung im Jahr $1980^{2}$, da sie in Artikel 19 Nr. 8 ein Recht auf eine kontaminationsfreie Umwelt und den Umweltschutz als Staatsziel enthält und das Recht auf eine kontaminationsfreie Umwelt durch eine beschränkte Verfassungsbeschwerde absichert ${ }^{3}$. Eine wichtige Station dieser zweiten Entwicklungsstufe ist das Gesetz Nr. 19.300: das Umweltrahmengesetz (Gesetz über die allgemeinen Grundlagen der Umwelt). Es wurde im März 1994 bekannt gemacht.

* Für die Unterstützung bei der Erstellung des Manuskripts möchte ich mich bei Herrn Prof. Dr. Klaus Lange und Herrn Dr. Arndt Schmehl bedanken.

1

Die Feststellung muss ergänzt werden. Während der 60er bis Anfang der 70er Jahre hatte das Umweltvölkerrecht eine entscheidende Bedeutung. Die Worte Umwelt oder Umweltrecht wurden noch nicht benutzt, aber der chilenische Staat schloß bereits wichtige umweltvölkerrechtliche Verträge ab, die noch als nationales Recht in Kraft sind, z.B. das Abkommen von Washington zum Schutz der Pflanzen, Tiere und der natürlichen Sehenswürdigkeiten von Amerika, das seit 1967 rechtsverbindlich ist. Infolge dieses Abkommens wurde das chilenische Naturschutzgebietssystem weiterentwickelt.

2 Die chilenische Verfassung wurde im März 1981 wirksam. Wegen ihres antidemokratischen Ursprungs wurde sie immer sehr stark kritisiert. Nach der Niederlage von Pinochet in dem Plebiszit vom 5. Oktober 1988 einigten sich die politischen Parteien (die rechten Parteien und die MitteLinks-Koalition) darüber, die Verfassung zu ändern. Im Juli 1989 wurde die geänderte Verfassung durch eine Volksabstimmung akzeptiert. Trotzdem dauern bis heute die politischen Streitigkeiten über die noch überlebenden autoritären Institutionen an.

3 Als Vorbilder der chilenischen Verfassung galten die portugiesische Verfassung von 1976 [Art. 66. (1) Jeder hat das Recht auf eine menschenwürdige, gesunde und ökologisch ausgewogene Umwelt, und ist verpflichtet für ihre Erhaltung Sorge zu tragen] und die spanische Verfassung von 1978 [Art. 45. (1) Alle haben das Recht, eine der Entfaltung der Persönlichkeit förderliche Umwelt zu genießen, sowie die Pflicht, sie zu erhalten]. 
Diese Arbeit enthält einen Überblick über das chilenische Umweltrecht. Dazu werden zunächst die Begriffe Umwelt und kontaminationsfreie Umwelt analysiert. Danach werden die Merkmale des Rechts auf eine kontaminationsfreie Umwelt sowie der Verfassungsbeschwerde näher dargelegt. Die grundrechtlichen Grenzen des Umweltschutzes und der Schutz der Umwelt als Staatsziel werden skizziert und anhand einiger Beispiele ein Blick auf die Realität des Umweltschutzes geworfen.

\section{Umweltbezogene Vorschriften der chilenischen Verfassung}

Der erste Absatz des Artikels 19 Nr. 8 der chilenischen Verfassung lautet:

„Art. 19. Die Verfassung sichert allen Menschen zu: (...)

Nr. 8. Das Recht auf das Leben in einer kontaminationsfreien Umwelt. Es ist die Pflicht des Staates darüber zu wachen, dass dieses Recht nicht verletzt wird, und Sorge für die Erhaltung der Natur zu tragen"“".

Der zweite Absatz des Artikels 19 Nr. 8 enthält eine Ermächtigung, die Ausübung bestimmter Rechte oder Freiheiten zum Schutz der Umwelt einzuschränken. Die Vorschrift des Art. 19 Nr. 8 muss im Zusammenhang mit dem Art. 20 Abs. 2 gesehen werden. Diese Norm eröffnet die Verfassungsbeschwerde, wenn das Recht auf eine kontaminationsfreie Umwelt infolge einer willkürlichen und gesetzwidrigen Handlung verletzt wird und dies durch eine bestimmte Behörde oder Person verursacht wurde. Hinsichtlich des privaten Eigentumsrechts verfügt Art. 19 Nr. 24 Abs. 2, dass privates Eigentum eingeschränkt werden kann, wenn die Erhaltung der Umwelt dies erfordert. Die zitierten Vorschriften begründen das chilenische Umweltverfassungsrecht ${ }^{5}$.

\section{Der Umweltbegriff}

Der Gesetzgeber hat in Art. 1 des Umweltrahmengesetzes Nr. 19.300 beschlossen, dass das Recht auf das Leben in einer kontaminationsfreien Umwelt und die Erhaltung der Natur u.a. durch dieses Gesetz geregelt werden. Hierbei ist wichtig, dass einerseits das Recht der Personen geschützt wird und andererseits die Natur als solches. Diese Vorschrift hätte als Gerüst des chilenischen Umweltrechts gelten können. Aus den Regelungen, die das Gesetz

Art. 19: La Constitución asegura a todas las personas: Nr. 8 El derecho a vivir en un medio ambiente libre de Contaminación. Es deber del Estado velar para que este derecho no sea afectado y tutelar la preservación de la naturaleza.

5

Durch diesen Begriff werden die Verfassungsbestimmungen erfasst, die einen unmittelbaren Umweltbezug aufweisen. 
enthält, hätte sich theoretisch das moderne chilenische Umweltrecht entwickeln sollen ${ }^{6}$, aber die Novellierung des Umweltrechts hat nur zum Teil stattgefunden.

Was das Umweltrahmengesetz Nr. 19.300 effektiv neu regelt, ist die Einführung der Umweltverträglichkeitsprüfung, der Umwelthaftungsklage und der neuen Umweltstaatsverwaltung. Natürlich erzeugen solche neuen Institutionen positive Auswirkungen auf das Recht auf die kontaminationsfreie Umwelt und insbesondere auf die natürliche Umwelt. Jedoch enthält dieses Gesetz nur wenige Instrumente, die das Recht auf eine kontaminationsfreie Umwelt unmittelbar garantieren, oder die bei der Verwirklichung des Staatziels unmittelbar helfen ${ }^{7}$.

\section{Umwelt als Rechtsobjekt}

Das Umweltrahmengesetz Nr. 19.300 enthält in Art. 2 eine Reihe von allgemeingültigen umweltrechtlichen und technischen Begriffen ${ }^{8}$. Der Begriff der Umwelt wird darin definiert als ,globales System, das aus natürlichen und künstlichen Elementen physikalischer, chemischer oder biologischer Natur sowie soziokulturellen Elementen und deren Interaktionen, in ständiger Modifikation durch die Aktivitäten der Menschen und der Natur, besteht, und das die Existenz und die Entwicklung des Lebens in seinen vielfältigen Formen steuert und unterstützt" ${ }^{\text {"9 }}$.

6

„Theoretisch“ deshalb, weil Art. 1 des Umweltrahmengesetzes alle anderen alten gesetzlichen Umweltnormen in Kraft gelassen hat. Er lautet nämlich: „Das Recht auf das Leben in einer kontaminationsfreien Umwelt, die Erhaltung der Natur... werden durch die Normen dieses Gesetzes reguliert, unbeschadet anderer gesetzlicher Umweltbestimmungen“. Der Gesetzgeber hat durch diese Gesetzgebungstechnik die alte und ungeeignete Umweltrechtsordnung unberührt gelassen. Dabei wurde eine vorzügliche Chance versäumt, um das chilenische Umweltrecht neu zu koordinieren und von altem Ballast zu befreien.

Z.B. die Umwelthaftungsklage ist nur bei erheblichen Umweltschäden gerechtfertigt. In diesem Fall legt das Umweltrahmengesetz fest, dass nur eine beschränkte Gruppe aktiv legitimiert ist (keine Popularklage). Zuletzt wird die Entschädigungsmöglichkeit nur auf die immer schwierige Wiederherstellung begrenzt. Unter solchen Voraussetzungen wäre die normale zivilrechtliche Haftungsklage effektiver.

Art. 2 des Umweltrahmengesetzes hat als Schwachpunkt, dass er die notwendigen technischen und umweltrechtlichen Begriffe nicht definiert. Z.B. die Standardrichtlinien von allgemeinen und bekannten Regeln der Technik, des Standes der Technik sowie des Standes von Wissenschaft und Technik sind umweltrechtliche Begriffe, welche der chilenische Gesetzgeber einfach vergessen hat. J. Bermúdez, Estudio de los conceptos técnico-jurídicos del Derecho ambiental, Revista de Derecho Administrativo Económico de la Pontificia Universidad Católica de Chile, Vol. II Nr. 2 Jul-Dec 2000, S. 459 ff.

Medio Ambiente: el sistema global constituido por elementos naturales y artificiales de naturaleza física, química o biológica, socioculturales y sus interacciones, en permanente modificación por la acción humana o natural y que rige y condiciona la existencia y desarrollo de la vida en sus 
Das bloße Lesen der obigen Gesetzesvorschrift könnte zu der Annahme führen, dass in diesem Zusammenhang die Umwelt in ihrer Gesamtheit gemeint sei ${ }^{10}$. Es ist klar, dass die Umwelt ein komplexes System ist ${ }^{11}$, dessen Schutz nicht auf einzelne Bestandteile beschränkt werden darf ${ }^{12}$. Eine wörtliche Auslegung des gesetzlichen Begriffes würde jedoch zu einem falschen Verständnis der Aufgaben des Staates gegenüber dem Menschen und seiner Umwelt führen. In diesem Kontext ist $\mathrm{zu}$ beachten, dass der gesetzliche Umweltbegriff nur den Anteil umfasst, der auf den menschlichen Lebensbereich Einfluss nimmt $^{13}$. Das ist von Bedeutung für die Auslegung des Art. 19 Nr. 8 der chilenischen Verfassung, da Art. 1 des Umweltrahmengesetzes auch das Recht auf die kontaminationsfreie Umwelt reguliert. Hierfür wurde der Begriff ,,angrenzendes Umfeld“ (Entorno adyacente ${ }^{14}$ vorgeschlagen, d.h. die Umwelt, die einen unmittelbaren Einfluss auf das Leben eines Einzelnen hat. Das angrenzende Umfeld enthält nicht das ganze Ökosystem, sondern den Teil, der in der Umgebung der betroffenen Menschen liegt und auf sie einwirkt. Das angrenzende Umfeld wird nicht so beschränkt betrachtet wie die Rechtssphäre, die durch andere Rechte geschützt wird. Im Gegenteil bedeutet das angrenzende Umfeld, als Rechtssphäre des Rechts auf eine kontaminationsfreie Umwelt, einen eigenen und differenzierten Bereich, der weiter und größer ist als andere Rechtsbereiche wie das Recht auf Leben und körperliche Unversehrtheit ${ }^{15}$. Andere Auslegungen würden dem effet utile des Rechts auf die kontaminationsfreie Umwelt zuwider laufen.

Die Rechtsprechung geht stillschweigend von dem Begriff ,,angrenzendes Umfeld“ aus. Der Fall Albornoz gegen Sociedad Maderera de Aysén ist dafür ein gutes Beispiel: Eine Holzfirma (,Sociedad Maderera de Aysén“) hatte eine größere Anzahl von Bäumen des Urwaldes, der zum Besitz der Familie Albornoz gehört, ohne Genehmigung der Forstverwaltung

múltiples manifestaciones. Für das deutsche Wort „Umwelt“ wird im Spanischen der Begriff „Medio Ambiente“ verwendet. Dieses Doppelwort enthält eine Tautologie, die Worte Medio und Ambiente haben für sich allein die gleiche Bedeutung, nämlich „Umwelt“.

$\S 2$ Abs. 1 UVPG und $\S 3$ Abs. 2 BImSchG könnten zu demselben Verständnis führen. Diese beiden Vorschriften stellen einen extensiven Umweltbegriff dar. Vgl. M. Kloepfer, Umweltrecht, 2. Aufl. 1998, S. 19-20.

11 Deshalb wurde über einen „schillernden Begriff der Umwelt“ gesprochen. In diesem Sinne W. Maus, Juristische Arbeitsblätter 1979, S. 288.

Vgl. F. Niederstadt, Ökosystemschutz durch Regelungen des öffentlichen Umweltrechts, 1998, S. $66 \mathrm{ff}$.

13 Hierbei stellt sich die Frage, ob der Begriff der anthropozentrischen oder der ökozentrischen Auffassung des Umweltschutzes entspricht, da die kommentierte Bestimmung über das Leben in seinen multiplen Formen spricht und nicht über Menschen.

Einzelheiten bei J. Bermúdez, Sistemas Sancionadores de Protección ambiental („Sanktionssysteme im Umweltrecht“) Dissertation, Madrid 1998, S. 422 ff. Existenz, sondern auch eine menschenwürdige Ausgestaltung. Deswegen sollte ein Anspruch auf die Umwelt auf dieser Basis aufgebaut werden. 
abgeholzt. Die Kläger haben ihren Wohnsitz in San Bernardo (in Hauptstadtnähe, in der geografischen Mitte Chiles). Ihr Wald liegt hingegen in Cohyaique (Südchile) mehr als $1000 \mathrm{~km}$ entfernt von San Bernardo. Der Oberste Gerichtshof des Staates (Corte Suprema de Justicia) hat in seinem Urteil von 19.01.1993 entschieden: „Nur derjenige hat das Recht eine Verfassungsbeschwerde zu erheben, der durch eine Handlung unmittelbar beeinträchtigt wurde. Dieses Recht besaßen die Beschwerdeführer nicht, da sie, gemäß ihrer eigenen Aussage, ständig in San Bernardo wohnen und deswegen durch eine Kontamination ${ }^{16}$ der Umwelt in der Region von Aysén, mehr als $1000 \mathrm{~km}$ entfernt, nicht unmittelbar beeinträchtigt wurden. “" ${ }^{17}$ Der Umweltverfassungsbeschwerde der Familie wurde deshalb vom Obersten Gerichtshof Chiles nicht stattgegeben. Die Beeinträchtigung des privaten Eigentumsrechts wurde hingegen anerkannt.

Der Umweltbegriff enthält einen zweiten Aspekt, welcher eine Erweiterung des Anwendungsbereiches darstellt. Die Umwelt im rechtlichen Sinn umfasst nicht nur die natürlichen Lebensgrundlagen, sondern auch die künstlichen und soziokulturellen Elemente. Da der größte Teil der Bevölkerung in Ballungsgebieten wohnt, darf ein gesetzlicher Umweltbegriff nicht nur die natürlichen Elemente Luft, Wasser und Boden (restriktiver Umweltbegriff) erfassen, sondern sollte auch die menschlich geprägten Elemente enthalten. Infolgedessen werden die soziokulturellen Elemente der Umwelt wie Denkmale, Kunstwerke und archäologische Stätten auch umweltrechtlich geschützt. In der Praxis werden diese Elemente auch im Verwaltungsverfahren der Umweltverträglichkeitsprüfung berücksichtigt.

\section{Umwelt, Kontamination und umweltrechtliche Standards}

Art. 19 Nr. 8 der Verfassung gewährleistet das Recht auf eine „kontaminationsfreie Umwelt“. Die wörtliche Interpretation dieser konstitutionellen Norm führte bis zu der unerreichbaren Utopie des Lebens in einer Umwelt, die keinerlei Kontamination enthält. Deshalb war es nötig, das Konzept zu präzisieren. Art. 2 Umweltrahmengesetz definiert „kontaminationsfreie Umwelt“ folgendermaßen: ,... dass die Kontamination in Konzentration und Dauer der Exposition unterhalb derjenigen liegen sollte, die ein Risiko für die

Der Oberste Gerichtshof hat hier einen weiten Begriff von Kontamination angewandt, da er unter Kontamination auch Zerstörung versteht. Ein Jahr später hat das Umweltrahmengesetz allerdings einen engeren Begriff definiert. Gemäß Art. 2 wird Kontamination definiert als in der Umwelt befindliche Stoffe, Elemente, Strahlen oder ihre Kombination, die die Grenzwerte überschreiten.

17

Corte Suprema 19.01.1993: El derecho a recurrir de protección solamente lo tiene quien es directamente afectado por el hecho, calidad que no tienen los recurrentes quienes según sus propias declaraciones viven en San Bernardo y no podrían ser afectados con una eventual contaminación del medio ambiente ocurrida en la región de Aysén distante más de mil kilómetros de la Región Metropolitana. 
Gesundheit der Menschen, die Lebensqualität der Bevölkerung, die Erhaltung der Natur oder die Erhaltung der überkommenen Umwelt darstellt“" ${ }^{18}$. Dieses Konzept wird kritisiert, weil es unvereinbare Rechtsgüter umfasst, die unterschiedliche Umweltstandards erfordern. Die Gesundheit der Personen, die Lebensqualität der Bevölkerung, die Erhaltung der Natur und die Erhaltung der überkommenen Umwelt verlangen unterschiedliche Kontaminationsgrenzwerte, z.B. verlangt die Lebensqualität höhere Umweltstandards als die bloße Gesundheit der Menschen ${ }^{19}$. Infolgedessen muss geklärt werden, unter welchen Umständen das Recht auf eine kontaminationsfreie Umwelt gesichert werden muss, selbst wenn eine Kontamination kein Risiko für die Gesundheit der Menschen oder für die Lebensqualität darstellt. Zusammenfassend kann man feststellen, dass die Gesundheit der Menschen und andere Rechte, wie das Recht auf Leben und körperliche Unversehrtheit oft näher liegen als das Recht auf eine kontaminationsfreie Umwelt ${ }^{20}$.

Das skizzierte Problem hat für das chilenische Umweltverfassungsrecht eine andere Bedeutung als z.B. für das deutsche. Da das Grundgesetz kein Grundrecht auf eine kontaminationsfreie, bzw. menschenwürdige Umwelt enthält, erfolgt eine weite Auslegung anderer Grundrechte um eine derartige Anspruchsposition zu erhalten. So wird aus dem Recht auf Leben und körperliche Unversehrtheit z.B. ein ökologisches Existenzminimum abgeleitet $^{21}$. Je weiter der Anwendungsbereich eines Rechts ist (z.B. Leben und körperliche Unversehrtheit), desto bessere Möglichkeiten bestehen, schädigende Einwirkungen auf die Umwelt zu vermeiden $^{22}$. Besteht dagegen, wie in Chile, auch zugleich ein ausdrückliches Umweltgrundrecht, so ergibt sich die Frage, inwieweit die Rechtssphäre der verschiedenen Rechte (auf eine kontaminationsfreie Umwelt und Leben und körperliche Unversehrtheit $^{23}$ ), unterschieden werden kann. Es gibt vermutlich Fälle, in denen sich die beiden

Medio Ambiente Libre de Contaminación: aquél en el que los contaminantes se encuentran en concentraciones y períodos inferiores a aquéllos susceptibles de constituir un riesgo a la salud de las personas, a la calidad de vida de la población, a la preservación de la naturaleza o a la conservación del patrimonio ambiental.

19

Hier wird ein enger Gesundheitsbegriff angewandt. Im Gegensatz dazu hat die World Health Organization die Gesundheit im weiteren Sinne definiert: Gesundheit ist der Zustand des vollständigen körperlichen, geistigen und sozialen Wohlbefindens und nicht nur des Freiseins von Krankheiten und Gebrechen.

J. Bermúdez, El derecho a vivir en un medio ambiente libre de contaminación. Revista de Derecho de la Universidad Católica de Valparaíso 2000, Nr. 21, S. 9.

21 D. Cansier, Gefahrenabwehr und Risikovorsorge im Umweltschutz und der Spielraum für ökonomische Instrumente, NVwZ 1994, S. 642; auch M. Kloepfer, Zum Grundrecht auf Umweltschutz, 1978, S. 27.

In gleichem Sinne B. Bock, Umweltschutz im Spiegel von Verfassungsrecht und Verfassungspolitik, 1990, S. $126 \mathrm{ff}$.

23

Art. 19 Nr. 1 La Constitución asegura a todas las personas: El derecho a la vida y a la integridad física y psíquica de la persona. 
Rechtsbereiche überlagern ${ }^{24}$. Der effet utile des Rechts auf eine kontaminationsfreie Umwelt verlangt allerdings eine Auslegung, die ihm einen eigenständigen Anwendungsbereich sichert.

Die Anwendung des Begriffes „kontaminationsfreie Umwelt“ verlangt einerseits die räumliche Zuordnung auf ein bestimmtes Gebiet und andererseits die Festlegung von Grenzwerten der Kontamination. In diesem Zusammenhang unterscheidet das Umweltrahmengesetz Nr. 19.300 zwei Arten von Normen: die Umweltqualitätsnormen und die Emissionsnormen $^{25}$. Die Umweltqualitätsnormen beschäftigen sich mit zwei Aspekten: dem Einwirkungsort oder Gebiet der Kontamination und den akzeptablen Ausmaßen der Kontamination $^{26}$. In den Emissionsnormen sind die zulässigen Maximalwerte des Ausstoßes, der z.B. von einer Industrieanlage ausgehen darf, festgelegt ${ }^{27}$. Das förmliche Verfahren, das die Schaffung der Normen reguliert, umfasst die Beteiligung von Privatpersonen und die Einholung von Sachverständigengutachten. Für die Entscheidungen über Grenzwerte ist das Präsidialamt (Ministerio Secretaría General de la Presidencia) zuständig. Die Umweltqualitätsnormen müssen alle fünf Jahre überprüft werden.

Der Erlass einer Umweltqualitätsnorm bzw. Emissionsnorm wird üblicherweise sehr scharf diskutiert, da die Entscheidungen stark politisch begründet sind. Selbstverständlich ist die Grenzwertfestsetzung eine Frage die nicht nur wissenschaftlicher oder technischer, sondern auch politischer Art ist, vor allem wenn die Sachverständigen keine konkreten Maßstäbe vorschlagen können ${ }^{28}$. Probleme treten auf, wenn von wissenschaftlichen und technischen Sachverständigen und Gremien Grenzwerte empfohlen wurden, die dann bei den politischen Entscheidungen nicht berücksichtigt werden. Eine Grenzwertfestsetzung verlangt einen Kompromiss zwischen Naturschutz und ökonomischem Wachstum, auch in Verantwortung für die künftigen Generationen.

Vgl. M. Böhm, Abschied vom Vorsorgeprinzip im umweltbezogenen Gesundheitsschutz, in: $K$. Lange (Hrsg.), Gesamtverantwortung statt Verantwortungsparzellierung im Umweltrecht, 1997, S. $44 \mathrm{ff}$.

Die Umweltqualitätsnormen und die Emissionsnormen sind vergleichbar mit den Normkategorien, die sich in den Verwaltungsvorschriften des $\$ 48$ BImSchG befinden. Die Außenwirkung von solchen Normen ist jedoch ein Thema, das die chilenische Fachliteratur noch nicht bearbeitet hat.

Die Umweltqualitätsnormen (normas de calidad ambiental) entsprechen etwa den Immissionen, die in $\S 3$ Abs. 2 BImSchG aufgeführt sind.

27 Vgl. § 3 Abs. 3 BImSchG.

28 R. Hendler, Umweltrechtliche Grenzwerte in der Gerichts- und Verwaltungspraxis, DÖV 1998, S. $482 \mathrm{ff}$. 


\section{Grenzwertfestlegung für die Schwebstaubbelastung in Santiago de Chile}

Ein typisches Beispiel für die Problematik der Grenzwertfestlegung ist die Schwebstaubbelastung in der chilenischen Hauptstadt. Mit ca. 5,5 Millionen Einwohnern ist Santiago die größte Stadt Chiles und befindet sich unter den 10 am stärksten kontaminierten Städten der Welt. Ein chaotisches öffentliches Verkehrssystem, die räumliche Konzentrierung der größten Industriebetriebe des Landes und eine für die Luftzufuhr ungünstige Topographie zählen zu den Ursachen des immensen Umweltproblems ${ }^{29}$.

Für die Immissionswerte von Schwebstaub PM10 $0^{30}$ wurde in der chilenischen Luftqualitätsnorm eine Massenkonzentration von $150 \mu \mathrm{g} / \mathrm{m}^{3}$ in 24 Stunden als Grenzwert für gute Luftqualität festgelegt ${ }^{31}$. Dieser Wert liegt schon relativ hoch, die World Health Organization (WHO) empfiehlt einen Grenzwert von $50 \mu \mathrm{g} / \mathrm{m}^{3}$. Die folgende Tabelle zeigt das Bewertungsschema für die Luftqualität in Santiago de Chile. Laut einer Studie der chilenischen Umweltverwaltung (Comisión Nacional del Medio Ambiente, CONAMA) wurden z.B. im Jahr 1998 an 120 von 334 Tagen Schwebstaubwerte über $195 \mu \mathrm{g} / \mathrm{m}^{3}$ gemessen $^{32}$.

Bewertungsschema zur Luftqualität in Santiago de Chile bezogen auf die Schwebstaubkonzentration

\begin{tabular}{|c|l|c|}
\hline PM 10 $\boldsymbol{\mu g} / \mathbf{m}^{\mathbf{3}}$ in 24 h & Bewertung & Warnstufe \\
\hline $0-150$ & Gut & - \\
\hline $150-195$ & Befriedigend & - \\
\hline $195-239$ & Schlecht & Alerta \\
\hline $240-284$ & Kritisch & Preemergencia \\
\hline $285-329$ & Gefährlich & Preemergencia \\
\hline 330 & Sehr gefährlich & Emergencia \\
\hline
\end{tabular}

Unter den gegenwärtigen Umständen wäre der von der WHO vorgeschlagene Umweltstandard von PM10 $50 \mu \mathrm{g} / \mathrm{m}^{3}$ in einer Stadt wie Santiago de Chile natürlich unrealistisch ${ }^{33}$. Doch welche kurz- und längerfristigen Kosten für die Umwelt und die Gesundheit der

Detaillierte Information unter: www.conama.cl (chilenische Umweltverwaltung) und www.terram.cl (chilenische NGO).

30

PM10 = Particulate Matter $\leq 10 \mu \mathrm{m}$, also Feinstaub mit einem Durchmesser bis $10 \mu \mathrm{m}$.

31

Die chilenische Luftqualitätsnorm wurde durch das Präsidialamt (,Ministerio Secretaría General de la Presidencia“) bestimmt: Decreto Supremo Nr. 59/1998.

32 Improvement of the Forecast of air quality and the knowledge of the local meteorological conditions in the metropolitan region, www.conama.cl/rm/.

33

Die Topographie der chilenischen Hauptstadt und die thermische Inversion (Thermal Inversion) im Winter schaffen einen Kesseleffekt, so dass die Luftverunreinigung, insbesondere der Schwebstaub, nicht entweichen kann. 
Bevölkerung hat diese lockere Grenzwertfestsetzung? Bereits jetzt sind die Krankenhäuser im Winter mit älteren Menschen und Kindern überfüllt, die an starken Atembeschwerden und kontaminationsbedingten Infektionskrankheiten leiden. Solche rechtspolitischen Fragen können in dieser Arbeit allerdings nicht beantwortet werden.

Der dreistufige staatliche Notfallplan enthält die folgenden Schutzmaßnahmen um die Schwebstaubfreisetzung zu reduzieren:

Alerta

- Fahrverbot für 40\% der Autos ohne Katalysator (4 Endziffern von 10).

- Heizverbot mit Holz, Holzkohle und Sägemehl für Kamine die keine zwei Filtersysteme enthalten.

Preemergencia

- Fahrverbot für 60\% der Autos ohne Katalysator (6 Endziffern von 10) und für 20\% der Autos mit Katalysator (2 Endziffern von 10).

- Heizverbot mit Holz, Holzkohle und Sägemehl für alle Kamintypen.

- Betriebsverbot für Anlagen die mehr als $32 \mathrm{mg} / \mathrm{m}^{3}$ PM10 freisetzen.

Emergencia

- Fahrverbot für $80 \%$ der Autos ohne Katalysator (8 Endziffern von 10) und für $40 \%$ der Autos mit Katalysator (4 Endziffern von 10)

- Heizverbot mit Holz, Holzkohle und Sägemehl für alle Kamintypen

- Betriebsverbot für Anlagen die mehr als $28 \mathrm{mg} / \mathrm{m}^{3} \mathrm{PM} 10$ freisetzen.

Diese kurzfristige Notbremse ist natürlich nicht ausreichend. Die Kontamination der Hauptstadt muss durch langfristige Maßnahmen verringert werden. Es stellt sich die Frage, inwieweit die entscheidende Behörde eine Chance hat entsprechende Veränderungen durchzusetzen. 


\section{Das konstitutionelle Recht auf eine kontaminationsfreie Umwelt und sein Schutz durch die Verfassung}

\section{Recht auf eine kontaminationsfreie die Umwelt als konstitutionelles Recht}

Die chilenische Verfassung enthält nur einen einzigen Katalog von Rechten, die in Art. 19 aufgeführt sind ${ }^{34}$. Darin liegt ein formeller Unterschied zu anderen Verfassungen (z.B. der spanischen Verfassung), die differenzierte Kategorien oder Listen für Grundrechte und soziale bzw. ökonomische Rechte enthalten ${ }^{35}$. Die konstitutionellen Rechte, die in den 26 Absätzen des Art. 19 enthalten sind, lassen sich aber in verschiedene Gruppen einordnen. Innerhalb der konstitutionellen Rechte unterscheidet die chilenische Verfassung danach, ob sie durch die Verfassungsbeschwerde garantiert sind oder nicht. Das Recht auf eine kontaminationsfreie Umwelt wird durch die Verfassungsklage geschützt.

$\mathrm{Ob}$ das Recht auf eine kontaminationsfreie Umwelt in seinem Wesen ein Grundrecht ist, ist eine Frage, die die chilenische Fachliteratur nicht beantworten musste. Alle Rechte, die in dem Kapitel III der Verfassung festgelegt sind, werden als „konstitutionelle Rechte“ bezeichnet $^{36}$. Der Ausdruck „Grundrecht“ ist in diesem Zusammenhang fremd. In der chilenischen Verfassungsgeschichte wurde immer von den Begriffen „Verfassungsgarantie“ und „konstitutionelle Rechte“ gesprochen (z.B. in den vorherigen chilenischen Verfassungen von 1833 und 1925). Deswegen ist der verbreitete Begriff „Grundrecht“ in der chilenischen Rechtskultur nicht bekannt.

Diese Feststellung gilt auch für das Recht auf eine kontaminationsfreie Umwelt, welches sich im Kapitel III über „Konstitutionelle Rechte und Pflichten“ befindet. Außerdem wird die Bezeichnung ,das Recht auf“ in Art. 19 Nr. 8 und in Art. 20 Abs. 2 ausdrücklich verwendet. Danach stellt das Recht auf eine kontaminationsfreie Umwelt eindeutig ein „konstitutionelles Recht“ dar ${ }^{37}$. Sein Anwendungsbereich wird allerdings nicht klar festge-

Kapitel III über Konstitutionelle Rechte und Pflichten umfaßt nur fünf Artikel (19 bis 23). Art. 19 umfasst 26 Absätze.

Art. 45 über das Recht auf eine angemessene Umwelt befindet sich im Abschnitt I „Von den Grundrechten und Pflichten“, Kapitel III Von den Leitsätzen der Sozial- und Wirtschaftspolitik. Vgl. L. Parejo Alfonso, Umweltschutz und Umweltrecht in Spanien, DVB1 1992, S. 1271.

Art. 5 Abs. 2 der chilenischen Verfassung legt als Bindung der Staatsgewalten fest ,die wesentlichen Rechte, die von der Natur des Menschen ausgehen“. Die Staatsgewalten haben die Pflicht diese Rechte, welche sich in der Verfassung und in den Staatsverträgen befinden, einzuhalten und zu fördern. Hier stellen sich die Fragen ob diese „,wesentlichen Rechte“ ganz oder teilweise den konstitutionellen Rechten entsprechen und ob die Rechte, die sich in den Staatsverträgen befinden, Verfassungsrang haben. Diese Fragen gehen über das Ziel dieser Arbeit hinaus.

Im spanischen Umweltverfassungsrecht ist umstritten, ob der Art. 45 der spanischen Verfassung als subjektives öffentliches Recht bezeichnet werden kann oder als bloßes Verfassungsprinzip. 
legt, vor allem weil Art. 19 Nr. 8 ein Recht auf das „Leben“ in einer kontaminationsfreien Umwelt garantiert. Die Verwendung dieses Wortes führte dazu, dass einige Autoren annahmen, dass das Recht auf eine kontaminationsfreie Umwelt eine Erweiterung des Rechts ,auf Leben“ (Art. 19 Nr. 1) sei $^{38}$.

Es stellt sich nun die Frage, ob ein konstitutionelles Recht als Grundrecht bezeichnet werden kann, wenn es durch die Verfassungsbeschwerde geschützt wird ${ }^{39}$. Art. 20 regelt die Verfassungsbeschwerde und enthält in Abs. 1 eine Auflistung einiger Rechte aus Art. 19, bei denen Verfassungsklage gewährt wird. Die Rechte auf Leben, Gleichheit, privates Eigentum, Freiheitsrechte usw. sind alle durch die sog. allgemeine Verfassungsbeschwerde gesichert. Die chilenische Verfassung unterscheidet sich von vielen anderen Verfassungen aber durch Art. 20 Abs. 2. Dieser widmet sich dem verfassungsmäßigen Schutz des Rechts auf eine kontaminationsfreie Umwelt, das durch eine spezielle Verfassungsbeschwerde eingeklagt werden kann. In der chilenischen Verfassung könnte demnach das Recht auf eine kontaminationsfreie Umwelt als Grundrecht eingestuft werden. Allerdings ist der Fall etwas komplexer, wie die folgenden Überlegungen zeigen.

\section{Allgemeine und spezielle Verfassungsbeschwerde}

Zur Einforderung des gerichtlichen Schutzes seiner konstitutionellen Rechte kann der Bürger eine Verfassungsbeschwerde erheben, die dann in einem schnellen Verfahren bearbeitet wird. Die Klage wird beim Appellationsgerichtshof (Corte de Apelaciones) eingereicht. Zuständig für die zweite Instanz ist der Oberste Gerichtshof des Staates (Corte Suprema de Justicia $)^{40}$. Eine Rechtswegerschöpfung wie z.B. in Deutschland und Spanien ist nicht notwendig.

Es stellt sich nun die Frage, warum die Verfassung in Art. 20 Abs. 2 eine spezifische Vorschrift für die Umweltverfassungsbeschwerde enthält. Es handelt sich um eine Unterscheidung zwischen der ,allgemeinen“ und der „,speziellen“ bzw. umweltrechtlichen Verfassungsbeschwerde. Der Unterschied liegt in den jeweiligen Anspruchsvoraussetzungen.

Vgl. F. Velasco Caballero, El medio ambiente en la Constitución: ¿derecho público subjetivo y/o principio rector?, Revista Andaluza de Administración Pública Nr. 19, Jul.-Sept., 1994, S. 77.

Z.B. E. Soto, El derecho a vivir en un medio ambiente libre de contaminación: su contenido esencial, Revista Gaceta Jurídica Nr. 151, Jan. 1993, S. 22 ff. Dagegen J. Bermúdez (Fn. 20).

39

Vgl. F. Velasco Caballero (Fn. 37).

40

Die Kontrolle der Verfassungsmäßigkeit ist in Chile verteilt zwischen den Verfassungsgerichthof (Tribunal Constitucional) und den Obersten Gerichtshof (Corte Suprema). Die sog. ex ante Normenkontrolle obliegt dem Verfassungsgerichtshof. Dagegen ist der Oberste Gerichtshof für die zweite Instanz der Verfassungsbeschwerde und für die ex post Normenkontrolle zuständig. 
Gemäß Art. 20 Abs. 1 ist eine allgemeine Verfassungsklage nur zulässig, wenn konstitutionelle Rechte bedroht, beeinträchtigt oder zunichte gemacht werden. Diese sind in der Liste des Artikels 20 Abs. 1 enthalten ${ }^{41}$. In diesem Fall kann der Kläger sein Recht gegen willkürliche oder gesetzwidrige Handlungen schützen. Diese Vorschrift enthält geringere Voraussetzungen für eine Klage. Die Klage bedarf keiner ausführlichen Begründung, allerdings muss eine Klagefrist von 15 Tagen eingehalten werden.

$\mathrm{Ob}$ die Verfassungsbeschwerde gegen Handlungen von Privatpersonen anwendbar ist oder nur gegen Handlungen des Staates, ist eine Frage, die seit längerem die Rechtsprechung beschäftigt hat. Da Art. 20 Abs. 1 allgemeine und geringe Voraussetzungen fordert, hat der chilenische Oberste Gerichtshof entschieden, dass eine weite Auslegung des Art. 20 Abs. 1 anzuwenden ist. Deshalb ist die Verfassungsbeschwerde auch zulässig, wenn eine Privatperson eine willkürliche oder gesetzwidrige Handlung verursacht hat. Hierzu muss man wissen, dass die Frage der „Drittwirkung“ von Grundrechten im chilenischen Verfassungsrecht nicht problematisch ist. Die konstitutionellen Rechte schützen als Abwehr- oder Freiheitsrechte gegenüber dem Staat, aber sie gelten auch als Rechtspositionen gegenüber Privatpersonen und müssen in Beziehungen zwischen nichtstaatlichen Rechtssubjekten eingehalten werden.

Die allgemeine Verfassungsbeschwerde ist gerechtfertigt, wenn folgende drei Bedingungen erfüllt sind:

a) Eine Handlung muss nachweisbar sein. Dies kann eine aktive Tätigkeit (positive Aktion) oder Unterlassung/Versäumnis (negative Aktion) sein.

b) Willkürlichkeit oder Gesetzwidrigkeit. Die Handlung muss willkürlich oder gesetzwidrig sein. Hierbei ist die Konjunktion „oder“ wichtig, es handelt sicht also nicht um eine verbindende Voraussetzung, sondern um eine alternative.

c) Bedrohungen, Beeinträchtigungen oder Zerstörungen der konstitutionellen Rechte. Die Verfassung bietet Schutz aufgrund einer Beschwerde nicht nur, wenn das Recht verletzt wird, sondern auch, wenn dieses bedroht wird. Das ist entscheidend für die Prävention einer Rechtsverletzung.

Art. 20 Abs. 1: El que por causa de actos u omisiones arbitrarios o ilegales sufra privación, perturbación o amenaza en el legítimo ejercicio de los derechos y garantías establecidos en el artículo 19 , números $1^{\circ}, 2^{\circ}, 3^{\circ}$ inciso cuarto, $4^{\circ}, 5^{\circ}, 6^{\circ}, 9^{\circ}$ inciso final, $11^{\circ}, 12^{\circ}, 13^{\circ}, 15^{\circ}, 16^{\circ}$ en lo relativo a la libertad de trabajo y al derecho a su libre elección y libre contratación, y a lo establecido en el inciso cuarto, $19^{\circ}, 21^{\circ}, 22^{\circ}, 23^{\circ}, 24^{\circ}$ y $25^{\circ}$ podrá ocurrir por sí o por cualquiera a su nombre, a la Corte de Apelaciones respectiva, la que adoptará de inmediato las providencias que juzgue necesarias para restablecer el imperio del derecho y asegurar la debida protección del afectado, sin perjuicio de los demás derechos que pueda hacer valer ante la autoridad o los tribunales correspondientes. 
Im Gegensatz dazu wird die sog. ,,spezielle“ oder Umweltverfassungsbeschwerde in Art. 20 Abs. 2 eingeschränkt. Der zweite Absatz des Artikels sagt Folgendes aus:

„Es gibt auch die Möglichkeit der Verfassungsklage im Fall des Artikels 19 Nr. 8, wenn das Recht auf das Leben in einer kontaminationsfreien Umwelt durch eine willkürliche und illegale Handlung gefährdet ist, an der eine bestimmte Behörde oder Person schuldig ist ${ }^{*}{ }^{42}$.

Die Unterschiede zwischen „allgemeiner“ und „spezieller“ Verfassungsbeschwerde sind erheblich. In dieser Hinsicht sind folgende Aspekte zu beachten:

a) Die Handlung, die das Recht auf das Leben in einer kontaminationsfreien Umwelt beeinträchtigt, kann nur eine aktive Handlung sein. Die Rechtsprechung hält sich direkt an die wörtliche Auslegung, sodass in diesem Fall die Klage allein gegen aktive Handlungen zulässig ist, nicht aber gegen Unterlassungen. Gemäß Art. 19 Nr. 8 erfüllt der Staat mit dem Rechtsschutz gegen solche Aktivitäten dabei seine Pflicht als Umweltschützer. Diese Aufgabe ist schwierig von Fällen abzugrenzen, bei denen die Staatsverwaltung selbst durch ihr Handeln das Recht auf eine kontaminationsfreie Umwelt verletzt. Wenn diese Pflichtwidrigkeit aus einer Unterlassung des Staates, die Umwelt zu schützen resultiert, könnte das verletzte Grundrecht auf eine kontaminationsfreie Umwelt durch die spezielle Verfassungsbeschwerde nicht eingeklagt werden.

b) Willkürliche und gesetzwidrige Handlung. Hier steht die Konjunktion ,und“, d.h. es handelt sich um eine verbindende Voraussetzung. Die Rechtsprechung hat diese doppelte Voraussetzung jedoch sehr flexibel ausgelegt und in der Regel betrachtet der Gerichtshof eine Handlung des Staates bzw. Privatpersonen schon dann als willkürlich und gesetzwidrig, wenn sie nur gesetzwidrig ist ${ }^{43}$.

c) Es muss sich um eine „Effektivverletzung“ handeln. Im Art. 20 Abs. 2 sind nicht die drei Möglichkeiten - Bedrohungen, Beeinträchtigungen oder Zerstörungen - der Handlung gegeben, sondern diese Norm verlangt eine Verletzung des Rechts. Das heißt, dass eine bloße Bedrohung nicht ausreichend wäre ${ }^{44}$. Gemäß der Rechtsprechung ist diese Effektivverletzung nur bei Folgen einer schädigenden Handlung gegeben, aber nicht bei Fällen von Umweltschäden infolge einer Unterlassung.

Art. 20 Inciso 2: "Procederá, también, el recurso de protección en el caso del $\mathrm{N}^{\circ} 8$ del artículo 19, cuando el derecho a vivir en un medio ambiente libre de contaminación sea afectado por un acto arbitrario e ilegal imputable a una autoridad o persona determinada".

In einigen Fällen von allgemeinen Verfassungsbeschwerden (willkürlich oder gesetzwidrig) hat die Rechtsprechung entschieden, dass eine gesetzmäßige Handlung als willkürlich angesehen werden soll, wenn sie den Verhältnismäßigkeitsgrundsatz nicht beachtet hat. Allerdings wäre der Umweltverfassungsbeschwerde nur stattzugeben, wenn die andere Voraussetzung (gesetzwidrige Handlung) ebenfalls erfüllt wird. recurso constitucional de protección sobre materia ambiental en Chile, Revista de Derecho de la Universidad Católica de Valparaíso, Nr. 13, 1989, 1990, S. 175 ff. 
d) Die Verletzung des Rechts wurde durch die Handlung einer bestimmten Behörde oder Person verursacht. Der Kläger muss in seiner Verfassungsbeschwerde die Personen oder Behörden ausdrücklich bezeichnen, die ihn in seinem Recht auf eine kontaminationsfreie Umwelt verletzt haben. In diesem Kontext ergibt sich ein Problem bei solchen Fällen, bei denen die Verletzung des Rechts durch verschiedene Verursacher bewirkt wird oder, im Gegenteil, keine Handlung eines bestimmten Verursachers vorliegt. Da die Umweltbelastungen normalerweise von Privatpersonen verursacht werden, soll die Verfassungsbeschwerde sowohl gegen Handlungen von staatlichen als auch von nichtstaatlichen Personen zulässig sein ${ }^{45}$. Aus der Sicht der deutschen Grundrechtstheorie wäre diese Auffassung fraglich, hier tritt die Schutzpflichtkomponente des Grundrechts in $\mathrm{Kraft}^{46}$. Dagegen hat sich die chilenische Lehre in eine andere Richtung entwickelt. Diese Doppelauffassung des konstitutionellen Rechts erlaubt, dass ein subjektives Recht wie das Recht auf eine kontaminationsfreie Umwelt an eine traditionelle Verfassung angepasst werden kann und durch die Verfassungsbeschwerde geschützt wird..

Ein wichtiger Punkt ist es, den Umweltbegriff auf das Recht auf eine kontaminationsfreie Umwelt zu begrenzen. Deshalb schützt die Verfassungsklage nur das Grundrecht (auf eine kontaminationsfreie Umwelt), nicht aber die ganze Umwelt. Wird die Umwelt als geschütztes Rechtsgut betrachtet, ist sie durch andere Rechtsinstrumente geschützt (z.B. die Umwelthaftungsklage). Hier hat sich die Rechtsprechung auf eine anthropozentrische Auslegung festgelegt. Was durch die Verfassungsbeschwerde garantiert wird, ist das Recht auf eine kontaminationsfreie Umwelt. Allerdings steht dieses Recht nur natürlichen Personen zu. Deswegen kann eine juristische Person (z.B. ein Naturschutzverband) nicht für sich selbst eine Verfassungsbeschwerde erheben ${ }^{47}$.

Vor zwei Jahren wurde im Parlament eine Vorlage zur Änderung der einschlägigen Verfassungsbestimmung eingebracht. Der Gesetzentwurf beabsichtigte, die Verfassungsbeschwerde des Art. 20 Abs. 2 hinsichtlich des Grundrechts der Menschen auf eine kontaminationsfreie Umwelt an die allgemeine Verfassungsbeschwerde anzugleichen. Als Folge dieses Gesetzentwurfes könnten die Bürger eine Verfassungsbeschwerde auch dann einreichen, wenn das Recht auf eine kontaminationsfreie Umwelt durch eine Unterlassung beeinträchtigt wird. Diese Initiative zur Verfassungsänderung hatte keinen Erfolg, da sie für den Staat eine immense Ausweitung seiner Aufgaben bedeutet hätte. Wie Kloepfer erläutert hat: „Das Verfassungsrecht selbst kann als Rechtsregelung nicht Flugzeuge geräuscharm fliegen lassen, Industrieabgase unschädlich machen, umgekippte Gewässer wieder mit

Vgl. M. Kloepfer (Fn. 21), S. 19.

46

47

Vgl. M. Böhm, Der Normmensch, 1996, S. $100 \mathrm{ff}$.

Z.B. die Entscheidung des Obersten Gerichthofs vom 4. Januar 1994. 
Leben erfüllen oder Erosionsflächen begrünen (...) Die Verfassung ist eben nicht eine säkularisierte Form des Allmächtigen “48.

\section{Umweltschutz als Grundrechtsschranke ${ }^{49}$}

Die Maßnahmen zum Schutz der Umwelt können zweifellos eine direkte Auswirkung auf andere Grundrechte haben. Gewerbefreiheit und Grundeigentum werden häufig durch Umweltschutzmaßnahmen berührt. Die Frage ob die Umwelt einen Vorrang vor anderen Rechten bzw. Rechtsgütern genießt, wird durch Art. 19 Nr. 8 Abs. 2 und Art. 19 Nr. 24 Abs. 2 der chilenischen Verfassung beantwortet.

Es ist allgemein anerkannt, dass nur der Gesetzgeber die Schrankensetzungskompetenz besitzt. In diesem Zusammenhang lässt sich die Gesetzesvorbehaltsklausel aus mehreren Bestimmungen der chilenischen Verfassung ableiten ${ }^{50}$. Diese Vorbehaltsklauseln können ausdrückliche oder implizite Klauseln sein $^{51}$, die aber immer in Verbindung mit einem spezifischen Recht stehen. Daraus läßt sich ableiten, dass die Schrankensetzungskompetenz des Gesetzgebers eine spezifische Ermächtigung bei jedem Recht voraussetzt. Im Gegensatz dazu ermächtigt Art. 19 Nr. 8 Abs. 2 zu einer generellen Schrankensetzungskompetenz in bezug auf alle anderen Rechte, mit dem Ziel, die Umwelt zu schützen. Art. 19 Nr. 8 Abs. 2 lautet: „Das Gesetz kann spezifische Einschränkungen für die Ausübung bestimmter Rechte oder Freiheiten zum Schutz der Umwelt festsetzen“" ${ }^{\circ 2}$.

Warum enthält die chilenische Verfassung diese generelle Ermächtigung des Gesetzgebers? Hierzu wird eine effektivitätsorientierte Auslegung vorgeschlagen, da die Verfassung keine überflüssige oder zwecklose Vorschrift enthält. In diesem Zusammenhang wird zunächst darauf hingewiesen, dass die obige Vorschrift eine Grundrechtseinschränkung zulässt, um die „Umwelt“ zu schützen. D.h. es wird nicht das Verhältnis zwischen verschiedenen konstitutionellen Rechten und dem Recht auf eine kontaminationsfreie Umwelt (Grund-

48

49

50

Z.B. Art. 19 Nr. 12 - Meinungs-, Informations-, Pressefreiheit; Nr. 15 - Vereinigungsfreiheit; Nr. 21 - Gewerbefreiheit, usw. Die Gesetzesvorbehaltsklausel umfasst formelle und materielle Aspekte. Der formelle Aspekt betrifft die Kompetenz, Schranken zu setzen. In diesem Zusammenhang verbietet Art. 61 Abs. 2 die Gesetzgebungsermächtigung vom Parlament zu allen Präsidenten (Die Ermächtigung des Präsidenten eine gesetzesvertretende Verordnung zu erlassen) u.a. hinsichtlich der Grundrechtsschranken.

51

52

M. Kloepfer (Fn. 21), S. 13.

In diesem Teil der Arbeit werden die Ausdrücke „Grundrecht“ und „konstitutionelles Recht“ als Synonym betrachtet.

Das gilt auch für das Grundgesetz. Vgl. R. Alexy, Theorie der Grundrechte, 1986, S. 263-264.

Art. 19 Nr. 8 Abs. 2: La Ley podrá establecer restricciones específicas al ejercicio de determinados derechos o libertades para proteger el medio ambiente. 
recht/Grundrecht) dargestellt, sondern zwischen Grundrecht und Umwelt oder subjektivem Recht und Rechtsgut. Es ist anzunehmen, dass die Bestimmung eine besondere Bedeutung für den Schutz der Umwelt hat. Der Gesetzgeber kann die Umwelt durch die Beschränkung entsprechender Grundrechte/Freiheiten schützen, er braucht dazu nicht die besondere Position oder das höhere Gewicht der Umwelt gegenüber anderen Rechten zu beweisen. Selbstverständlich muss das beschränkende Gesetz am Verhältnismäßigkeitsgrundsatz ${ }^{53}$ und Wesensgehalt der Grundrechte gemessen werden ${ }^{54}$. Aber die Ermächtigung ist so generell (über alle anderen Rechte) und so klar (Schutz der „Umwelt“), dass der Schutz der Umwelt einen besonderen Rang im chilenischen Verfassungsrecht erhält ${ }^{55}$.

Das Eigentumsrecht wird durch Art. 19 Nr. 24 garantiert. In Abs. 2 dieses Artikels wird der Gesetzgeber ermächtigt, Schranken des Eigentumsrechts zu setzen, wenn dies die Sozialbindung verlangt. Die Erhaltung der überkommenen Umwelt ist Bestandteil der Sozialbindung des Eigentumsrechts ${ }^{56}$.

\section{Schutz der Umwelt als Staatsziel}

Art. 19 besteht aus einem Verzeichnis der Rechte und enthält normalerweise nach jedem Recht das zugehörige Staatsziel. Der zitierte Art. 19 Nr. 8 ist ein gutes Beispiel dieser Regelungstechnik, da er das subjektive Recht und das Staatsziel enthält. Das erste Kapitel der Verfassung, in dem sich Art. 19 befindet, enthält dazu wichtige Leitprinzipien der Staatsorgane $^{57}$. Die Tätigkeit der Staatsorgane orientiert sich gemäß Art. 1 Abs. 4 am

J. Bermúdez (Fn. 20). Vgl. D. Cansier (Fn. 21).

Art. 19 Nr. 26 verbietet die Antastung der Grundrechte in ihrem Wesensgehalt.

Die traditionelle chilenische Fachliteratur über dieses Thema sagt, dass der Art. 19 Nr. 8 Abs. 2 eine besondere Wiederholung der Gesetzesvorbehaltklausel sei. Zudem habe der Verfassungsgeber diese Folgerung nicht vorgesehen. Doch ist die obige Auslegung eine plausible und begründete Interpretation dieser Vorschrift. Falls die chilenische Verfassung der Umwelt keine besondere Rolle zugedacht hätte, wäre Art. 19 Nr. 8 Abs. 2 einfach überflüssig. Diese Auslegung findet auch in der Rechtsprechung des BVerfG eine gute Begründung. Die Verfassungsbestimmungen können einen Bedeutungswandel erfahren, ,wenn in ihrem Bereich neue, nicht vorgesehene Tatbestände auftauchen oder bekannte Tatbestände durch ihre Einordnung in den Gesamtablauf einer Entwicklung in neuer Beziehung oder Bedeutung erscheinen“ (Urteile des Bundesverfassungsgerichts vom 1. Juli 1953, BVerfGE 2, 401, und 16. Juni 1954, BVerfGE 3, 422). Die zunehmende Beeinträchtigung der Umwelt und das gewachsene Umweltbewusstsein stellen eine solche Entwicklung dar, der in der Norminterpretation Rechnung zu tragen ist.

Art. 19 Nr. 24 Abs. 2: Sólo la ley puede establecer el modo de adquirir la propiedad, de usar, gozar y disponer de ella y las limitaciones y obligaciones que deriven de su función social. Esta comprende la conservación del patrimonio ambiental.

Kapitel I „Grundlagen der Institutionen des Staates“ (Bases de la Institucionalidad). Im Kapitel I befinden sich Prinzipien wie Legalität, Demokratie, republikanische Staatsform usw. 
Gemeinwohl $^{58}$. Dieses allgemeine Ziel erfährt eine Spezifizierung hinsichtlich der Umwelt in Art. 19 Nr. 8 Abs. 1: „Es ist die Pflicht des Staates, darüber zu wachen, dass dieses Recht (auf eine kontaminationsfreie Umwelt) nicht verletzt wird, und Sorge für die Erhaltung der Natur zu tragen“، ${ }^{59}$.

Eine naheliegende Schlussfolgerung ist, dass diese Umweltschutzpflicht den ganzen Staat verpflichtet, das heißt alle Staatsgewalten und nicht nur die Vollziehende Gewalt oder die Staatsverwaltung. Die Umweltschutzpflicht gilt für alle Staatsorgane gleichermaßen. Die Gesetzgebung kann subjektive Rechtspositionen unter Berücksichtigung dieser Pflicht festlegen $^{60}$. Allerdings besitzt die Verfassung gemäß Art. 6 auch unmittelbare Bindungswirkung, denn die Verwaltung und die Rechtsprechung müssen ihre Tätigkeit in Bezug auf diese konstitutionelle Pflicht ausüben ${ }^{61}$.

Hinsichtlich des Inhalts der Umweltschutzpflicht wird gefolgert, dass die Staatsorgane das Grundrecht auf eine kontaminationsfreie Umwelt schützen müssen. Gegen Verletzungen, die nicht in einer Unterlassung bestehen, ist, wie schon erwähnt, die spezielle Verfassungsbeschwerde anwendbar. Die Umweltschutzpflicht erfasst außerdem die Verantwortlichkeit für den Naturschutz (preservación de la naturaleza).

Diese doppelte Pflicht des Staates bedeutet einerseits, dass das Recht auf das Leben in einer kontaminationsfreien Umwelt gestärkt wird, und anderseits, dass der Schutz der Natur ein besonderes Staatsziel ist.

a) Stärkung des Rechts: Unstreitig ist, dass aus jedem Grundrecht eine Leistungspflicht für den Staat abgeleitet werden kann. Zum Teil wird diese Pflicht schon durch bloße Inaktivität des Staates erfüllt, z.B. bei den Freiheitsrechten. Im Gegensatz dazu soll das Recht auf eine kontaminationsfreie Umwelt durch eine aktive Leistung der Staatsorgane gesichert werden. Somit unterliegt der Staat dem Gebot, über das Umweltrecht zu

Art. 1 Abs. 4: El Estado está al servicio de la persona humana y su finalidad es promover el bien común, para lo cual debe contribuir a crear las condiciones sociales que permitan a todos y a cada uno de los integrantes de la comunidad nacional su mayor realización espiritual y material posible, con pleno respeto a los derechos y garantías que esta Constitución establece.

Dazu J. Bermúdez, Roles del Consejo de Defensa del Estado en la protección del medio ambiente: acción ambiental y recurso de protección en materia ambiental, Revista de Derecho de la Universidad Católica de Valparaíso, 1999 Nr. XX, S. 243.

60 Vgl. im deutschen Recht: H. Steiger, Verfassungsrechtliche Grundlagen, in: Arbeitskreis für Umweltrecht (Hrsg.), Grundzüge des Umweltrechts, 2. Aufl. 1997, S. 02/032.

61 Art. 6: Los órganos del Estado deben someter su acción a la Constitución y a las normas dictadas conforme a ella. Los preceptos de esta Constitución obligan tanto a los titulares o integrantes de dichos órganos como a toda persona, institución o grupo. La infracción de esta norma generará las responsabilidades y sanciones que determine la ley. 
wachen. Damit das Recht nicht verletzt wird, und um Grundrecht und Rechtsobjekt stärker zu verknüpfen, muss der Staat Umweltbeeinträchtigungen verhindern ${ }^{62}$.

b) Schutz der Natur als Staatsziel: Die konstitutionelle Vorschrift sagt, dass der Staat zuständig ist für die Erhaltung der Natur. Art. 2 des Umweltrahmengesetzes enthält einen Katalog mit umweltrechtlichen Begriffsbestimmungen. Innerhalb dieser Begriffe steht die „Erhaltung der Natur“. Sie ist folgendermaßen definiert: „Die gesamte Politik, Pläne, Programme, Normen und Handlungen müssen dazu bestimmt sein, die Bedingungen zu erhalten, die die Evolution und Entwicklung der Spezies und der Ökosysteme des Landes möglich machen “63 ${ }^{63}$ Diese Definition enthält weder konkrete Voraussetzungen dafür wann ein Handeln des Staates notwendig ist, noch Angaben über den Umfang der Maßnahmen. Dies wirft das Problem auf, dass der Staat unter Umständen inaktiv bleibt oder unzureichend handelt. In diesem Zusammenhang muss betont werden, dass das Staatsziel sich ausdrücklich auf die Erhaltung der Natur als unabhängiges Rechtsgut bezieht. Die Natur soll als Gesamtsystem geschützt werden, im Gegensatz zu der oben beschriebenen Auslegung bei der ausschließlich Naturschäden in Zusammenhang mit der Beeinträchtigung von Personen (angrenzendes Umfeld) berücksichtigt wurden.

\section{Schlußbemerkung: Realität des Umweltschutzes in Chile}

Chile ist ein Land der geografischen Extreme. Es ist mehr als $4000 \mathrm{~km}$ lang und maximal $450 \mathrm{~km}$ breit, von Norden nach Süden umfasst es subtropische Trockengebiete, gemäßigte Zonen und das eisige Feuerland. Entsprechend den verschiedenen Landschaften und Klimazonen findet man eine hohe Biodiversität. 19 Prozent der Oberfläche sind Naturschutzgebiet und deshalb rechtlich geschützt. Darunter befinden sich Nationalparks, Nationalreservate und Naturdenkmale.

Gleichzeitig hat Chile eine rasante wirtschaftliche Entwicklung hinter sich und galt bis vor wenigen Jahren als Vorbild für die anderen lateinamerikanischen Länder. Eine jährliche Wachstumsrate von 6 bis 7 \% zwischen 1987 und 1997 wurde als bester Beweis des chilenischen Wirtschaftwunders betrachtet. Es ist jedoch fraglich, ob dieses Wachstum einer nachhaltigen wirtschaftlichen Entwicklung entspricht. Die chilenische Wirtschaft basiert bis heute auf den Export von Rohstoffen. Mineralien, Holz, Meeresprodukte und landwirt-

In der Grundrechttheorie ist es heute anerkannt, dass die Grundrechte nicht nur als Abwehrrechte gelten, sondern auch sie der Schutzpflicht des Staates unterliegen. Die obige Bestimmung der chilenischen Verfassung enthält ausdrücklich diese objektive Schutzpflicht.

Preservación de la Naturaleza: el conjunto de políticas, planes, programas, normas y acciones, destinadas a asegurar la mantención de las condiciones que hacen posible la evolución y el desarrollo de las especies y de los ecosistemas del país. 
schaftliche Produkte stellen den größten Anteil der chilenischen Ausfuhrwaren dar. Dieses Exportmodell hat natürlich einen eindeutig negativen Einfluss auf die Umwelt. So fällt es schwer, über nachhaltige Entwicklung zu sprechen, wenn das Wirtschaftswachstum auf der Abholzung des Urwaldes beruht oder auf der expandierenden Lachszucht, die die Eutrophierung von vor kurzem noch sauberen Seen und Meeresbuchten nach sich zieht ${ }^{64}$.

Die Entwicklung des chilenischen Umweltrechts ist stark mit der wirtschaftlichen Entwicklung und mit den Außenhandelsbeziehungen des Landes verbunden. Da die Importländer umweltrechtliche Bedingungen stellen, müssen Länder wie Chile zumindest einige minimale Umweltstandards erreichen und nachweisen. Dieser Umstand hat wesentlich zur Entwicklung des chilenischen Umweltrechts beigetragen. Die beiden großen Instrumente des Umweltschutzes, die Umweltverfassungsbeschwerde und das Umweltrahmengesetz haben einen relativ hohen Standard im Vergleich zu Ländern in vergleichbaren Situationen erreicht, sie enthalten natürlich noch einige Schwächen. Das Recht auf das Leben in einer kontaminationsfreien Umwelt wird häufig als Teil des Rechts auf Leben und nicht als unabhängiges Recht interpretiert. Die Umweltverfassungsbeschwerde hat - wegen ihrer Beschränkungen - noch nicht die in sie gesetzten Erwartungen erfüllt. Ein Schwachpunkt des Umweltrahmengesetzes Nr. 19.300 ist außerdem der Mangel an Instrumenten zu dessen Durchsetzung $^{65}$. Der Bürger hat weiterhin nur begrenzte Möglichkeiten, den Staat zu Erfüllung seiner Pflichten zu bewegen. Die Entwicklung des chilenischen Umweltrechts hat allerdings gerade erst begonnen und hoffentlich noch viele Schritte vor sich.

Hinsichtlich des Fischereirechts ist zu bemerken, dass die Umweltverordnung für die Aquakultur im Dezember 2001 in Kraft getreten ist. protección del medio ambiente. Análisis Crítico. Estudios de Derecho Administrativo V, Instituto de Estudios de Derecho Administrativo, Mendoza/Argentinien 2001, S. 39. 